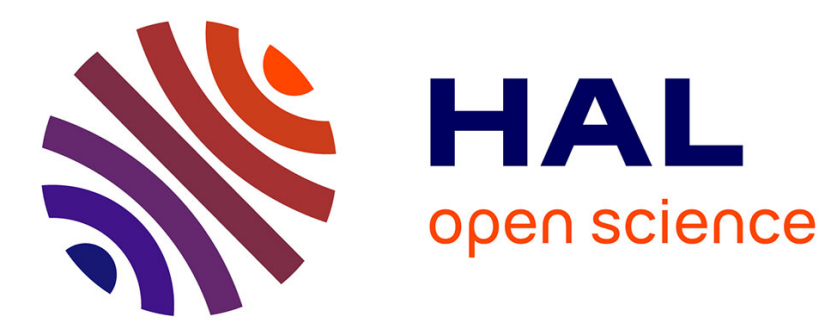

\title{
High-spin states in the five-valence-particle nucleus 213Po
}

\author{
A. Astier, M.-G. Porquet
}

\section{To cite this version:}

A. Astier, M.-G. Porquet. High-spin states in the five-valence-particle nucleus 213Po. Physical Review C, 2011, 83, pp.034302. 10.1103/PhysRevC.83.034302 . in2p3-00574162

\section{HAL Id: in2p3-00574162 https://hal.in2p3.fr/in2p3-00574162}

Submitted on 7 Mar 2011

HAL is a multi-disciplinary open access archive for the deposit and dissemination of scientific research documents, whether they are published or not. The documents may come from teaching and research institutions in France or abroad, or from public or private research centers.
L'archive ouverte pluridisciplinaire HAL, est destinée au dépôt et à la diffusion de documents scientifiques de niveau recherche, publiés ou non, émanant des établissements d'enseignement et de recherche français ou étrangers, des laboratoires publics ou privés. 


\title{
High-spin states in the five-valence-particle nucleus ${ }^{213} \mathrm{Po}$
}

\author{
Alain Astier and Marie-Geneviève Porquet \\ CSNSM, IN2P3/CNRS and Université Paris-Sud, F-91405 Orsay Campus, France
}

(Dated: January 5, 2011)

\begin{abstract}
Excited states in ${ }^{213} \mathrm{Po}$ have been populated using the ${ }^{18} \mathrm{O}+{ }^{208} \mathrm{~Pb}$ reaction at $85 \mathrm{MeV}$ beam energy and studied with the Euroball IV $\gamma$-multidetector array. The level scheme has been built up to $\sim 2.0 \mathrm{MeV}$ excitation energy and spin $I \sim 25 / 2 \hbar$ from the triple $\gamma$-coincidence data. Spin and parity values of several yrast states have been assigned from the $\gamma$-angular properties. The configurations of the yrast states are discussed using results of empirical shell-model calculations and by analogy with the neighbouring nuclei. The spin and parity values of several low-spin states of ${ }^{213}$ Po previously identified from the $\beta$-decay of ${ }^{213} \mathrm{Bi}$ are revised.
\end{abstract}

PACS numbers: $25.70 . \mathrm{Hi}, 27.80 .+\mathrm{w}, 23.20 . \mathrm{g}, 21.60 . \mathrm{Cs}$

\section{INTRODUCTION}

${ }^{213} \mathrm{Po}$ is a five-particle nucleus with two protons and three neutrons outside the doubly-magic core, ${ }^{208} \mathrm{~Pb}$. Thus one can expect its first excited states to come from a few spherical configurations mixed by the neutron-proton interactions. Some states issued from pure $\pi^{2}$ configurations on the one hand and from pure $\nu^{3}$ configurations on the other hand have been already identified in neighbouring nuclei: (i) the first excited states of ${ }^{210} \mathrm{Po}$ is a textbook example of residual interaction in a two-proton configuration, (ii) the properties of the three-neutron nucleus, ${ }^{211} \mathrm{~Pb}$, are well accounted for using shell model (SM) calculations [1].

It is worth pointing out that the SM predictions of nuclei lying above ${ }^{208} \mathrm{~Pb}$ are not numerous. For instance, large-scale SM calculations have been performed for the $N=126$ isotones, from $Z=84$ to $Z=94$, in the full $Z=82-126$ proton model space using the modified KuoHerling interaction [2] and semi-empirical shell model has been used to describe several nuclei close to ${ }^{208} \mathrm{~Pb}[3]$. Moreover the ground-state configuration of three oddodd nuclei, ${ }^{210} \mathrm{Bi}\left(\pi^{1} \nu^{1}\right),{ }^{212} \mathrm{Bi}\left(\pi^{1} \nu^{3}\right),{ }^{212} \mathrm{At}\left(\pi^{3} \nu^{1}\right)$, have been recently predicted using effective interactions derived from the CD-Bonn nucleon-nucleon potential [4], but there is no calculation dealing with many more valence nucleons. Indeed when adding a lot of protons and neutrons to the the doubly-magic core, ${ }^{208} \mathrm{~Pb}$, we reach the well-known region of octupole-deformed nuclei [5], which is ouside the scope of SM calculations because of a too large valence space. On the contrary, the study of the heavy Po nuclei with only two protons and many neutrons outside ${ }^{208} \mathrm{~Pb}$ allows us to investigate both the effective neutron-proton interactions and the configuration mixings, as these nuclei remain almost spherical.

In the present paper, we report on high-spin states in ${ }^{213} \mathrm{Po}$, which are identified for the first time. The level scheme has been built up to $\sim 2.0 \mathrm{MeV}$ excitation energy using high-fold $\gamma$ coincidences and $\gamma$-ray anisotropies have been measured to assign the spin values. The yrast states of ${ }^{213} \mathrm{Po}$ are discussed using results of empirical shell-model calculations and by analogy with the neighbouring nuclei.

\section{EXPERIMENTAL METHODS AND DATA ANALYSIS}

We have used the ${ }^{18} \mathrm{O}+{ }^{208} \mathrm{~Pb}$ reaction, the ${ }^{18} \mathrm{O}$ beam with an energy of $85 \mathrm{MeV}$ being provided by the Vivitron tandem of IReS (Strasbourg). A $100 \mathrm{mg} / \mathrm{cm}^{2}$ selfsupporting target of ${ }^{208} \mathrm{~Pb}$ was employed, which was thick enough to stop the recoiling nuclei. Due to this large thickness, the ${ }^{18} \mathrm{O}$ beam was stopped in the $\mathrm{Pb}$ target too and the incident energy covered a large range of values, from $85 \mathrm{MeV}$ down to the Coulomb barrier. The de-exciting $\gamma$-rays were recorded with the EUROBALL IV array consisting of 71 Compton-suppressed Ge detectors [6] (15 cluster germanium detectors placed in the backward hemisphere with respect to the beam, 26 clover germanium detectors located around $90^{\circ}, 30$ tapered single-crystal germanium detectors located at forward angles). Each cluster detector is composed of seven closely packed large-volume Ge crystals [7] and each clover detector consists of four smaller Ge crystals [8].

Events were recorded on tape when at least 3 unsuppressed Ge detectors fired in prompt coincidence. In this way, a set of $\sim 4 \times 10^{9}$ three- and higher-fold events were available for subsequent analysis. The main objective of the experiment was actually the study of the fusion-fission channel which leads to the production of the high-spin states of $\sim 150$ fragments, mainly located on the neutron-rich side of the valley of stability [9]. In addition, various transfer reactions have produced several Po isotopes. First of all, ${ }^{210,211,212,214}$ Po have been identified thanks to their known $\gamma$-ray yrast cascades [10]. Moreover many new $\gamma$ lines emitted by the two even-even ${ }^{212,214} \mathrm{Po}$ isotopes have been found to be strong enough in our data set to be precisely analyzed [11-13]. Then we have looked for the $\gamma$ lines emitted by ${ }^{213}$ Po.

Various procedures have been used for the offline analysis in order to fully characterize the excited levels of ${ }^{213} \mathrm{Po}$ (excitation energy, spin and parity values). Both multi-gated spectra and three-dimensional 'cubes' have been built and analyzed with the Radware package [14], starting from the X-rays of Po in order to identify the new transitions and to build the level scheme. 


\section{EXPERIMENTAL RESULTS}

Prior to this work, the excited states of ${ }^{213} \mathrm{Po}$ were studied from the $\beta$-decay of ${ }^{213} \mathrm{Bi}$ [15]. All the spin values assigned to these states are $7 / 2,9 / 2$ or $11 / 2$, because of the properties of the ground state of ${ }^{213} \mathrm{Bi}\left(\mathrm{I}^{\pi}=9 / 2^{-}\right)$. These excited states are not expected to be populated by a reaction induced by heavy ions, since their spin values are very close to the one of the ${ }^{213} \mathrm{Po}$ ground state $\left(\mathrm{I}^{\pi}=9 / 2^{+}\right)$. The last study of the $\beta$-decay of ${ }^{213} \mathrm{Bi}[16]$ has led to the identification of a new state, at $868.0 \mathrm{keV}$, which mainly de-excites towards the ground state. Its spin value, $\mathrm{I}=13 / 2$, was proposed on the basis of the comparison with results of semi-empirical shell-model calculations. In that case, the $868.0 \mathrm{keV} \gamma$-ray should be observed in the present work, as the first member of the yrast cascade.

In order to identify all the transitions depopulating the yrast states of ${ }^{213} \mathrm{Po}$, we have first looked into spectra gated by the $\mathrm{K}_{\alpha 1} \mathrm{X}_{\text {-ray }}{ }^{1}$ of Po. The spectrum of $\gamma$-rays in coincidence with two $\mathrm{K}_{\alpha 1} \mathrm{X}$-ray of Po displays, in addition to the transitions emitted by ${ }^{212} \mathrm{Po}[11,12]$, ${ }^{214} \mathrm{Po}$ [13], as well as ${ }^{210,211} \mathrm{Po}[10]$, three new transitions at $289 \mathrm{keV}, 423 \mathrm{keV}$, and $646 \mathrm{keV}$. They are in

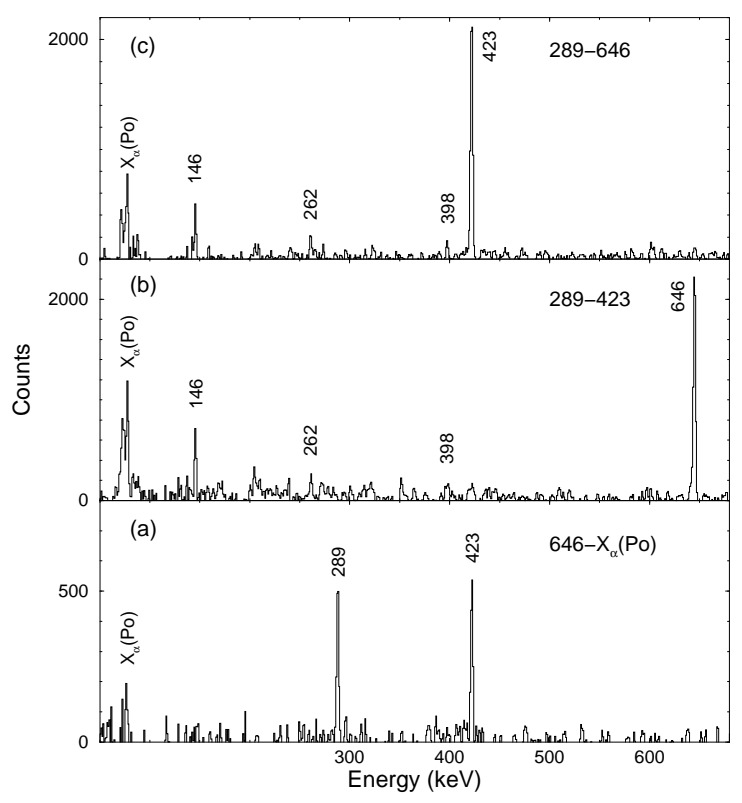

FIG. 1: Spectra of $\gamma$-rays in double coincidence with (a) the first transition of ${ }^{213} \mathrm{Po}(646 \mathrm{keV})$ and the $\mathrm{K}_{\alpha 1} \mathrm{X}$-ray of Po, (b) the $289 \mathrm{keV}$ and $423 \mathrm{keV}$ transitions, and (c) the $289 \mathrm{keV}$ and $646 \mathrm{keV}$ transitions.

\footnotetext{
1 Low-energy transitions, such as the X-rays of Po, are clearly observed in our work since we used a very low threshold for triggering the CFD discriminator of each Ge channel. This was allowed by the VXI electronic cards of the Euroball array. For that purpose, the lower thresholds of the 239 channels were carefully checked at the beginning of the data acquisition.
}

mutual coincidence (see, for instance, the three spectra of Fig. 1), but they are not in coincidence with the transitions emitted by the other Po isotopes. Thus they must belong to the yrast structure of ${ }^{213} \mathrm{Po}$, its population being expected in the reaction used in this work since all the neighbouring isotopes are observed. Then all the doublegated spectra involving these three transitions, the $K_{\alpha 1}$ X-rays, as well as all the weaker lines as those observed in the spectra of Fig. 1, have been analyzed. The level scheme built from all the observed coincidence relationships is shown in Fig. 2.

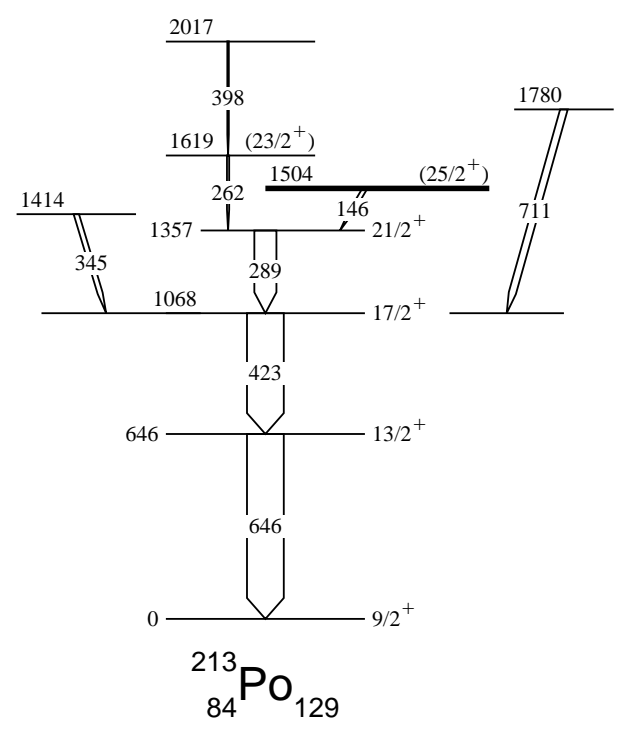

FIG. 2: Level scheme of ${ }^{213}$ Po determined in this work. The width of the arrows is representative of the relative intensity of the $\gamma$-rays. The level at $1504 \mathrm{keV}$ is likely a long-lived state (see Sect. IV).

We have gathered in Table I the properties of the transitions assigned to ${ }^{213} \mathrm{Po}$ from this work. The $\mathrm{R}_{A D O}$ angular anisotropy with respect to the beam axis has been analyzed to determine the multipole order of the most intense $\gamma$-rays, as done for the $\gamma$-rays of ${ }^{212,214} \mathrm{Po}$ obtained in the same experiment [11-13]. The results, given in Table I, allow us to determine the spin and parity values of the first yrast states. The $\mathrm{I}^{\pi}$ values of the $1504 \mathrm{keV}$ and $1619 \mathrm{keV}$ states, given in parenthesis, will be discussed in the following section.

As regards the $289 \mathrm{keV}$ transition, we have extracted its internal conversion coefficient using its intensity imbalances measured in spectra in double coincidence with the $146 \mathrm{keV}$ transition and either the $423 \mathrm{keV}$ or the $646 \mathrm{keV}$ transition. The obtained value, $\alpha_{\text {tot }}=0.15(5)$, corroborates the E2 multipolarity for the $289 \mathrm{keV}$ transition.

Lastly, we have estimated the cross section of the production of ${ }^{213} \mathrm{Po}$ in this ${ }^{18} \mathrm{O}+{ }^{208} \mathrm{~Pb}$ reaction, $\sigma \sim 0.3 \mathrm{mb}$. This is well lower than the value we have obtained for ${ }^{212} \mathrm{Po}, \sigma \sim 10-20 \mathrm{mb}[12]$ and about three times less 
TABLE I: Properties of the $\gamma$ transitions assigned to ${ }^{213} \mathrm{Po}$ from this work.

\begin{tabular}{ccccccc}
\hline \hline $\begin{array}{c}\mathrm{E}_{\gamma}{ }^{a} \\
\mathrm{keV}\end{array}$ & $\mathrm{I}_{\gamma}{ }^{b c}$ & $\mathrm{R}_{A D O}{ }^{d}$ & $\begin{array}{c}\mathrm{E}_{i} \\
\mathrm{keV}\end{array}$ & $\begin{array}{c}\mathrm{E}_{f} \\
\mathrm{keV}\end{array}$ & $I_{i}^{\pi}$ & $I_{f}^{\pi}$ \\
\hline 146.2 & $8(2)$ & & 1504 & 1357 & $\left(25 / 2^{+}\right)$ & $21 / 2^{+}$ \\
261.7 & $4.8(14)$ & & 1619 & 1357 & $\left(23 / 2^{+}\right)$ & $21 / 2^{+}$ \\
289.0 & $60(10)$ & $1.3(2)$ & 1357 & 1068 & $21 / 2^{+}$ & $17 / 2^{+}$ \\
344.5 & $15(5)$ & & 1413 & 1068 & & $17 / 2^{+}$ \\
398.1 & $3.5(12)$ & & 2017 & 1619 & & $\left(23 / 2^{+}\right)$ \\
422.8 & 100 & $1.18(10)$ & 1068 & 646 & $17 / 2^{+}$ & $13 / 2^{+}$ \\
645.6 & - & $1.25(10)$ & 646 & 0 & $13 / 2^{+}$ & $9 / 2^{+}$ \\
711.2 & $24(6)$ & & 1780 & 1068 & & $17 / 2^{+}$ \\
\hline \hline
\end{tabular}

${ }^{a}$ Uncertainties in transition energies are typically between 0.1 and $0.5 \mathrm{keV}$.

${ }^{b}$ Intensities measured in this experiment (i.e. with the requirement that a minimum of three unsuppressed Ge detectors fired in prompt coincidence) are normalized to the total intensity of the $\gamma$ transitions populating the $1068 \mathrm{keV}$ state.

${ }^{c}$ The number in parenthesis is the error in the last digit.

${ }^{d} \mathrm{R}_{A D O}=\mathrm{I}_{\gamma}\left(39.3^{\circ}\right) / \mathrm{I}_{\gamma}\left(76.6^{\circ}\right)$

than obtained for ${ }^{214} \mathrm{Po}, \sigma \sim 0.5-1 \mathrm{mb}$ [13]. At bombarding energies around and lower than $85 \mathrm{MeV}$, the ${ }^{18} \mathrm{O}$ projectiles may break up into ${ }^{14,12} \mathrm{C}+\mathrm{He}$. Using the kinematics of the exit channel leading to ${ }^{212} \mathrm{Po}$, we have shown [12] that the heavy partner of the break up, ${ }^{14} \mathrm{C}$, is emitted backward, implying that the production of ${ }^{212} \mathrm{Po}$ involves the fusion of the light partner, ${ }^{4} \mathrm{He}$, and the ${ }^{208} \mathrm{~Pb}$ target ${ }^{2}$. Thus we can infer that ${ }^{214} \mathrm{Po}$ is produced when the break up leads to ${ }^{12} \mathrm{C}$ as the heavy fragment. In such a scenario, excited states of ${ }^{213} \mathrm{Po}$ are likely produced from the levels of ${ }^{214} \mathrm{Po}$ having the highest energies and decaying by neutron emission.

Noteworthy is the fact that the abovementioned transition at $868 \mathrm{keV}$ has not been observed in our data set, it is correlated neither to the X-rays of Po nor to the transitions newly assigned to ${ }^{213} \mathrm{Po}$. Thus we do not confirm the previously proposed location of the $13 / 2^{+}$yrast state at $868 \mathrm{keV}$ [16]. A possible assignment of the $868 \mathrm{keV}$ state observed in the $\beta$-decay of ${ }^{213} \mathrm{Bi}$ will be discussed in the following section.

\section{DISCUSSION}

${ }^{213} \mathrm{Po}$ is a five-particle nucleus with two protons and three neutrons outside the doubly-magic core, ${ }^{208} \mathrm{~Pb}$. Thus one can expect that its first excited states come from the $\left(\pi h_{9 / 2}\right)^{2} \otimes\left(\nu g_{9 / 2}\right)^{3}$ shell-model (SM) configuration. The states issued from elementary SM configurations such as $\left(\nu g_{9 / 2}\right)^{3}$ and $\left(\pi h_{9 / 2}\right)^{2} \otimes\left(\nu g_{9 / 2}\right)^{1}$ can

2 The fusion of the heavy partner, ${ }^{14} \mathrm{C}$, and the target was proposed to be the main mechanism leading to the production of $\mathrm{Ra}$ isotopes in the ${ }^{18} \mathrm{O}+{ }^{208} \mathrm{~Pb}$ reaction at $92 \mathrm{MeV}$ [17]. be easily computed using the two-body empirical residual energies and the coefficients of fractional parentage (CFP) corresponding to the case of three nucleons [18]. For that purpose, the residual energies are extracted from the energies of the $\left(\nu g_{9 / 2}\right)^{2}$ multiplet (known in $\left.{ }^{210} \mathrm{~Pb}\right)$, the $\left(\pi h_{9 / 2}\right)^{2}$ multiplet (known in $\left.{ }^{210} \mathrm{Po}\right)$, and the $\left(\pi h_{9 / 2}\right)^{1} \otimes\left(\nu g_{9 / 2}\right)^{1}$ multiplet (known in $\left.{ }^{210} \mathrm{Bi}\right)$. The re-

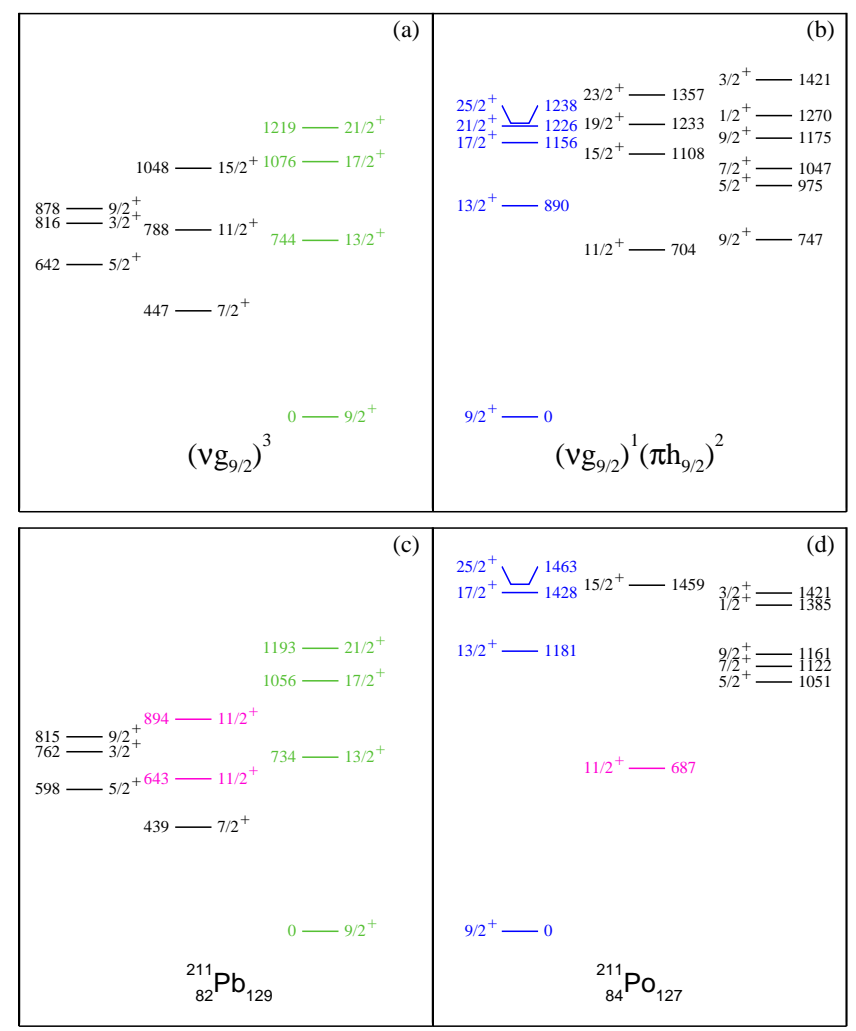

FIG. 3: (color on line) Top: Theoretical states belonging to the spherical $\left(\nu g_{9 / 2}\right)^{3}$ configuration (a) and $\left(\pi h_{9 / 2}\right)^{2} \otimes$ $\left(\nu g_{9 / 2}\right)^{1}$ configuration (b), computed using empirical interactions extracted from the two-nucleon neighbouring nuclei $\left({ }^{210} \mathrm{~Pb},{ }^{210} \mathrm{Po},{ }^{210} \mathrm{Bi}\right)$. The yrast states of the two sets are drawn in green and blue, respectively. Bottom: First excited states measured in the three-neutron nucleus, ${ }^{211} \mathrm{~Pb}[1,19]$ (c), and in the two-proton one-neutron nucleus, ${ }^{211}$ Po $[20,21]$ (d). The yrast states of the two nuclei are drawn in green and blue, respectively. The wave functions of the $11 / 2^{+}$states (drawn in magenta) contain a large component of the $\nu i_{11 / 2}$ orbital located just above the $\nu g_{9 / 2}$ one.

sults of the two calculations are given in Figs. 3(a) and $3(\mathrm{~b})$. The two groups of yrast states (drawn in green and blue) display similar features, those of the $\left(\nu g_{9 / 2}\right)^{3}$ configuration being slightly more compressed in energy. Moreover the $\left(\pi h_{9 / 2}\right)^{2} \otimes\left(\nu g_{9 / 2}\right)^{1}$ configuration leads to the maximum spin, $25 / 2^{+}$, the corresponding level is expected to be isomeric as it is located closely above the $21 / 2^{+}$state.

These predicted states compare very well with the results measured in two nuclei having these two elemen- 
tary SM configurations, ${ }^{211} \mathrm{~Pb}$ and ${ }^{211} \mathrm{Po}$ respectively (see Figs. 3(c) and $3(\mathrm{~d})$ ). ${ }^{211} \mathrm{~Pb}$ exhibits two $11 / 2^{+}$ states close in energy, their wave-functions are likely very mixed, one component from the $\left(\nu g_{9 / 2}\right)^{3}$ configuration and the other from the $\left(\nu g_{9 / 2}\right)^{2} \otimes\left(\nu i_{11 / 2}\right)^{1}$ one, the $\nu i_{11 / 2}$ orbital being located just above the $\nu g_{9 / 2}$ one. On the other hand, only one $11 / 2^{+}$state was observed in ${ }^{211} \mathrm{Po}$, its spectroscopic factor measured in the ${ }^{211} \mathrm{Po}(\mathrm{d}, \mathrm{p})$ reaction indicates that this state mainly comes from the $\left(\pi h_{9 / 2}\right)^{2} \otimes\left(\nu i_{11 / 2}\right)^{1}$ configuration [10], that means that another $11 / 2^{+}$state remains to be identified. As regards the yrast states, those of ${ }^{211} \mathrm{~Pb}$ are more compressed in energy than those of ${ }^{211}$ Po. Moreover the $25 / 2^{+}$state of ${ }^{211} \mathrm{Po}$ is a long-lived isomeric state $\left(\mathrm{T}_{1 / 2}=25.2(6) \mathrm{s}\right)$ deexciting through $\alpha$-decay [10], implying that the $21 / 2^{+}$ state is definitely higher in energy than the $25 / 2^{+}$state in this nucleus.

At this point, we can surmise that the $\left(\pi h_{9 / 2}\right)^{2} \otimes$ $\left(\nu g_{9 / 2}\right)^{3}$ configuration of ${ }_{84}^{213} \mathrm{Po}_{129}$ would display the following features:

- the lowest excitation mainly comes from the $\left(\nu g_{9 / 2}\right)^{3}$ configuration, that gives the $13 / 2_{1}^{+}$, $17 / 2_{1}^{+}$, and $21 / 2_{1}^{+}$states,

- the $25 / 2^{+}$state due to the $\left(\pi h_{9 / 2}\right)^{2} \otimes\left(\nu i_{11 / 2}\right)^{1}$ configuration is no longer a long-lived isomeric state decaying by $\alpha$ emission, as the $21 / 2_{1}^{+}$state is located below it.

This is in good agreement with the experimental result (see Fig. 2), provided that the $146 \mathrm{keV}$ transition is E2. It is worth recalling that we have got no direct information about its multipolarity, the only peculiar property of this $\gamma$-ray is the fact that no transition could be placed above the $1504 \mathrm{keV}$ state. That rules out the M1 case, as explained now. When taking into account the large value of the internal conversion coefficient $\left(\alpha_{\text {tot }}(146, \mathrm{M} 1)=\right.$ 3.8 ), the total intensity of the $146 \mathrm{keV}$ transition would amount to 40(10). Assuming that the intensity of a transition populating the $1504 \mathrm{keV}$ state is only $50 \%$ of its total decay, such a transition would give a line having an height around 700 counts in the spectrum of $\gamma$-rays in double coincidence with two yrast transitions, at variance with the observation (see the spectra in Fig. 1).

If the half-life of the $1504 \mathrm{keV}$ state is more longer than the time window used to delimit a coincidence event (300 ns in our experiment), the non-observation of the population of this state is obvious. Such a long halflife naturally occurs in case of an E2 transition with a low energy such as $146 \mathrm{keV}$, provided that the transition probability is slightly hindered: if $\mathrm{B}(\mathrm{E} 2)=0.1 W . u$., we obtain $\mathrm{T}_{1 / 2}(146 \mathrm{keV}, \mathrm{E} 2)=450 \mathrm{~ns}$. When assuming that the main configuration of the $21 / 2_{1}^{+}$state of ${ }^{213} \mathrm{Po}$ is not the same than that of the $25 / 2_{1}^{+}$state $\left[\left(\nu g_{9 / 2}\right)^{3}\right.$ versus $\left.\left(\pi h_{9 / 2}\right)^{2} \otimes\left(\nu i_{11 / 2}\right)^{1}\right]$, we expect a very hindered E2 transition probability. For instance, the yrast-state configurations of ${ }_{42}^{94} \mathrm{Mo}_{52}$ are known to shift from proton excitations to neutron ones, that gives $\mathrm{B}\left(\mathrm{E} 2 ; 8_{1}^{+} \rightarrow 6_{1}^{+}\right)=0.005$
W.u. [10]. In summary, the yrast states of ${ }^{213}$ Po identified in the present work are drawn in the right part of Fig. 4 using the same color code as those of Fig. 3 (green and blue).

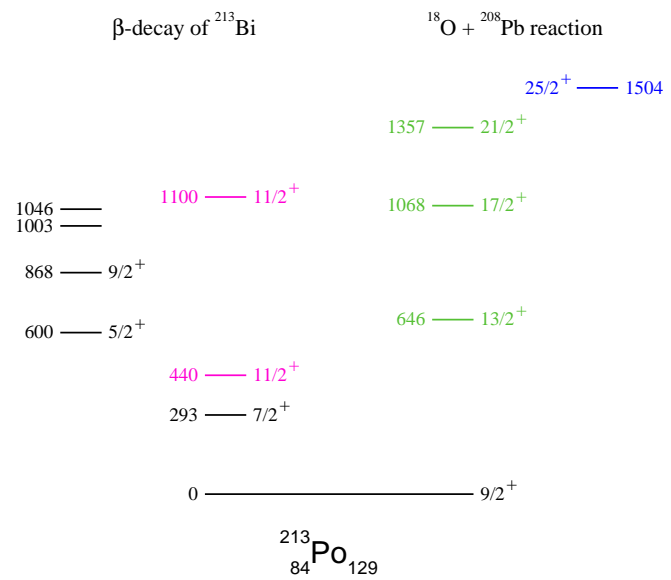

FIG. 4: (color on line) Right: High-spin levels of ${ }^{213}$ Po identified in the present work. Left: Low-spin states measured in the $\beta$-decay of the $9 / 2^{-}$ground state of ${ }^{213} \mathrm{Bi}$, the spin values (at variance with those assigned in ref. [16]) are discussed in the text. The color code is the same as that of Fig. 3.

As displayed in Fig. 3(a), the $\left(\nu g_{9 / 2}\right)^{3}$ configuration gives rise to several low-spin states which may be observed in the $\beta$-decay of the $9 / 2^{-}$ground state of ${ }_{83}^{213} \mathrm{Bi}[16]$. Moreover, as in ${ }^{211} \mathrm{~Pb}$, the $11 / 2^{+}$state of the $\left(\nu g_{9 / 2}\right)^{3}$ configuration can be strongly mixed to the state from the $\nu i_{11 / 2}$ orbital, leading to two $11 / 2^{+}$states having similar properties. To suggest the new spin values of the states measured in the $\beta$-decay which are drawn in the left part of Fig. 4, we made use of the following arguments:

- three states are directly populated by the $\beta$ decay, with similar logft values $(\sim 6.2)$ : the $9 / 2^{+}$ ground state and the excited states at $440 \mathrm{keV}$ and $1100 \mathrm{keV}[16]$.

- the $\beta$-decay of the $9 / 2^{-}$ground state of ${ }_{85}^{211}$ At populates two states of ${ }^{211} \mathrm{Po}_{127}$ with similar log ft values $(\sim 5.9)$ : the $\nu g_{9 / 2}$ ground state and the $\nu i_{11 / 2}$ excited state at $687 \mathrm{keV}[10]$.

- the $440 \mathrm{keV}$ and $1100 \mathrm{keV}$ states have similar decay schemes: one branch to the ground state and another one to the first excited state at $293 \mathrm{keV}$. Moreover the $1100 \mathrm{keV}$ state decays to the $440 \mathrm{keV}$ one.

- the first excited state of the $\left(\nu g_{9 / 2}\right)^{3}$ configuration is expected to have $\mathrm{I}^{\pi}=7 / 2^{+}$(see Fig. 3(a)).

Thus, combining results on the yrast states obtained in the present work and those on lower-spin states coming from the ${ }^{213} \mathrm{Bi} \beta$-decay [16], most of the states of 
the $\left(\nu g_{9 / 2}\right)^{3}$ configuration can be identified. As said above, two $11 / 2^{+}$states display similar features, that occurs when two unperturbed states are very close in energy, giving a maximum mixing of the two states (here, $\left[\left(\nu g_{9 / 2}\right)^{3}\right]_{11 / 2}$ and $\left.\left[\left(\nu g_{9 / 2}\right)^{2}\left(\nu i_{11 / 2}\right)^{1}\right]_{11 / 2}\right)$. Then we can assume that the energy of the $11 / 2^{+}$states of the two pure configurations is around half the sum of the two measured energies, i.e. $770 \mathrm{keV}$. This is in very good agreement with the predicted energy of the $11 / 2^{+}$state of the $\left(\nu g_{9 / 2}\right)^{3}$ configuration, $788 \mathrm{keV}$ (see Fig. 3(a)). As for the $\nu i_{11 / 2}$ orbital, it gives rise to many excited states having spin values in the range $1 / 2-27 / 2$, from the breaking of either the neutron pair or the proton pair of the $\left(\pi h_{9 / 2}\right)^{2}\left(\nu g_{9 / 2}\right)^{2}\left(\nu i_{11 / 2}\right)^{1}$ configuration. In any case, the yrast states lying above $1357 \mathrm{keV}$ (the $21 / 2^{+}$state from the $\left(\nu g_{9 / 2}\right)^{3}$ configuration) likely involve the $\nu i_{11 / 2}$ orbital.

\section{SUMMARY}

The ${ }^{18} \mathrm{O}+{ }^{208} \mathrm{~Pb}$ reaction at $85 \mathrm{MeV}$ beam energy was used to populate the high-spin states of ${ }^{213} \mathrm{Po}$. Their $\gamma$-decays were investigated using the Euroball IV array. The level scheme was built up to $\sim 2.0 \mathrm{MeV}$ excitation energy and spin $I \sim 25 / 2 \hbar$ from the triple $\gamma$-coincidence data. Spin and parity values of several yrast states were assigned from the $\gamma$-angular properties. Using results of empirical shell-model calculations and analogy with the neighbouring nuclei, the main configurations of the yrast states are proposed. The first excitations come from the three-neutron configurations, particularly the $\left(\nu g_{9 / 2}\right)^{3}$ one. The spin and parity values of several states previously known from the $\beta$-decay of ${ }^{213} \mathrm{Bi}$ were revised in order to account for the low-spin states expected in the $\left(\nu g_{9 / 2}\right)^{3}$ configuration. Shell model calculations using a realistic effective interaction derived from the CD-Bonn nucleon-nucleon potential have been recently performed in nuclei close to ${ }^{208} \mathrm{~Pb}$ [4], giving results in very good agreement with experiments. It would be worth extending them to nuclei having more valence nucleons, such as the five-particle nucleus, ${ }^{213}$ Po.

\section{Acknowledgments}

The Euroball project was a collaboration between France, the United Kingdom, Germany, Italy, Denmark and Sweden. We are very indebted to our colleagues involved in the EB-02/17 experiment devoted to the fission fragments, in which the present data on ${ }^{213}$ Po were recorded. We thank the crews of the Vivitron, as well as M.-A. Saettle for preparing the Pb target, P. Bednarczyk, J. Devin, J.-M. Gallone, P. Médina and D. Vintache for their help during the experiment.
[1] C.F. Liang, P. Paris, R.K. Sheline, Phys. Rev. C 58, 3223 (1998).

[2] E. Caurier, M. Rejmund and H. Grawe, Phys. Rev. C 67, 054310 (2003)

[3] G.D. Dracoulis et al., Phys. Rev. C 80, 054320 (2009) and references therein.

[4] L. Coraggio, A. Covello, A. Gargano and N. Itaco, Phys. Rev. C 80, 021305(R) (2009) and references therein.

[5] I. Ahmad and P. Butler, Ann. Rev. Nucl. Part. Sci. 43, $71(1993)$

[6] J. Simpson, Z. Phys. A358, 139 (1997).

[7] J. Eberth et al., Nucl. Instr. Meth. A 369, 135 (1996).

[8] G. Duchêne et al., Nucl. Instr. Meth. A 432, 90 (1999)

[9] M.-G. Porquet, Int. J. Mod. Phys. E13, 29 (2004).

[10] ENSDF data base, http://www.nndc.bnl.gov/ensdf/.

[11] A. Astier, P. Petkov, M.-G. Porquet, D.S. Delion, and P. Schuck, Phys. Rev. Lett. 104, 042701 (2010).

[12] A. Astier, P. Petkov, M.-G. Porquet, D.S. Delion, and P.
Schuck, Eur. Phys. J. A 46, 165 (2010).

[13] A. Astier and M.-G. Porquet, accepted for publication, Phys. Rev. C. (Dec 2010).

[14] D.C. Radford, Nucl. Instr. Meth. Phys. Res. A 361, 297 and 306 (1995).

[15] M.S. Basunia Nuclear Data Sheet 108, 633 (2007).

[16] G. Ardisson, V. Barci and O. El Samad, Phys. Rev. C $\mathbf{5 7}, 612$ (1998).

[17] D. Hojman et al., Phys. Rev. C 73, 044604 (2006).

[18] I. Talmi, Simple Models of Complex Nuclei, (Harwood Academic Publishers, 1993).

[19] G.J. Lane et al., Phys. Lett. B 606, 34 (2005).

[20] B. Fant, T. Lönnroth and V. Rahkonen, Nucl. Phys. A 355, 171 (1981).

[21] T.R. McGoram, G.D. Dracoulis, A.P. Byrne, A.R. Poletti, S. Bayer, Nucl. Phys. A 637, 469 (1998). 